\title{
Studies on Polyelectrolyte Solutions VII. Fast, Heterogeneous, and Slow Diffusion Modes of Poly(diallyl- $N$, $N$-dimethylammonium chloride) in Aqueous Alcoholic Salt Solvents
}

\author{
Wolfgang NiERLING and Eckhard NordmeIER ${ }^{\dagger}$ \\ Department of Physical Chemistry, University of Osnabrück, \\ 49069 Osnabrück, Germany
}

(Received September 24, 1996)

\begin{abstract}
Dynamic light scattering measurements were performed on aqueous alcoholic salt solutions of poly(diallyl$N, N$-dimethylammonium chloride) (PDADMAC, $M_{w}=2.5 \times 10^{5} \mathrm{~g} \mathrm{~mol}^{-1}$ ) in the semidilute regime, where the salts used were $\mathrm{NaCl}$ and $\mathrm{Na}_{2} \mathrm{SO}_{4}$. The results indicate the existence of three diffusive processes, a fast, heterogeneous, and a slow mode. The fast diffusion mode, $D_{\mathrm{fass}}$, is interpreted as the coupled motion of a polyion blob with its surrounding small ions. Such a blob contains in average $g$ segments which are connected to give a linear coil where the coil behaves as if it were isolated in a dilute solution. Experimentally, it is found that $g$ increases with increasing salt concentration and increasing alcoholic content. This indicates that the electrostatic segment-segment interactions become more and more screened the poorer the solvent quality is. The slow diffusion mode, $D_{\text {slow }}$, refers to the motion of large polyion clusters, having an internal ordered structure. These clusters can be as large as $300 \mathrm{~nm}$ or more. They are stabilized by intermolecular electrostatic interactions and become destroyed by strong hydrophobic interactions, that is when the degree of polyion dissociation converges versus zero, so that the polyions become neutral. At this limit $D_{\text {slow }}$ disappears and may become equal to $D_{\text {cop }}$, the cooperative diffusion coefficient of a semidilute solution of neutral polymers. The heterogeneous diffusion mode, $D_{\text {hetero }}$, is caused by the motion of parts of polyion chains that are not incorporated into the polyion cluster domains. Their total concentration is usually rather small so that an observation is often difficult. At one extreme, such a polyion part can be as small as a blob. Then there is no heterogeneous diffusion mode, that is we have $D_{\text {hetero }}=D_{\text {fast }}$. At the other extreme, $D_{\text {hetero }}$ may describe the motion of a complete single polyion chain whose dimensions may be as large as a cluster domain so that $D_{\text {hetero }}$ becomes equal $D_{\text {slow }}$. As a consequence, while $D_{\text {fast }}$ and $D_{\text {slow }}$ are nearly always present, $D_{\text {hetero }}$ may lie too near by $D_{\text {fast }}$ or $D_{\text {slow }}$, respectively, so that a separation becomes impossible. The simultaneous detection of all three modes is possible only by using the multiple tau technique. With the old correlators and the old conventional analysis methods a decomposition of the diffusion modes is very difficult if not impossible. Mostly one finds only one or two modes, which has lead to the erroneous idea, that there is an extraordinary-ordinary diffusion phase splitting. In reality, however, there is no splitting or phase transition. All three modes are present simultaneously where the only technical problem is their resolution.

KEY WORDS Polyelectrolyte / Poly(diallyl- $N, N$-dimethylammonium chloride) / Dynamic Light Scattering / Fast Diffusion Mode / Heterogeneous Diffusion Mode / Slow Diffusion Mode / Mixed Solvents /
\end{abstract}

Dynamic light scattering studies on polyelectrolytes solutions at low salt concentrations yield an interesting effect. There is a sudden drop of the diffusion coefficient, $D$, as the salt concentration, $c_{\mathrm{S}}$, is decreased below a critical value. ${ }^{1}$ That is, there are two diffusive modes, a relatively fast one of the order of some microseconds and a slow one of the order of $10^{3}-10^{4} \mu \mathrm{s}$. The effect is called the "extraordinary-ordinary" transition or the fast/slow diffusion mode splitting, where the fast mode is termed as ordinary and the slow mode as extraordinary. The ratio, $D_{\text {fast }} / D_{\text {slow }}$, can be as large as 100 and the onset and the strength of the splitting depends on various parameters. They are among others the absolute ionic strength, the polyion concentration, and the polyion charge density, respectively. In some cases, both modes become observable simultaneously, whereas in other cases only the slow mode is detectable. ${ }^{2,3}$ It should be pointed out that this slow mode is a characteristic of polyelectrolytes. Neutral polymers show also both a fast and a slow diffusion mode, but at significantly higher polymer concentrations. ${ }^{4}$ There $D_{\text {slow }}$ may be interpreted as the translational diffusion of intermolecularly entangled polymer chains ${ }^{4}$ or in terms of osmotic pressure fluctuations and viscoelasticity, ${ }^{5}$ while in the con-

\footnotetext{
† To whom correspondence should be addressed.
}

centration regime considered here polyion entanglements are unlikely.

The occurence of the fast/slow diffusion mode splitting was first reported in polylysine solutions by Lin et $a l .{ }^{1}$ Later it was also found in salt-free or low-salt solutions of DNA, ${ }^{6,7}$ t-RNA, ${ }^{8}$ bovine serum albumin (BSA), ${ }^{9}$ polystyrene sulfonate (PSS), ${ }^{10-12}$ polynucleosomes, ${ }^{13}$ polyadenylic acid, ${ }^{14}$ poly(acrylic acid) ${ }^{15}$ poly(methacrylic acid), ${ }^{16}$ and quaternized poly(vinylpyridine). ${ }^{17}$

The physical reason for the fast mode seems to be clear. At low polyion concentration, $D_{\text {fast }}$ decreases monotonically with increasing salt concentration $c_{\mathrm{S}}$. This behavior is typical for the coupled diffusion of polyions with counterions. ${ }^{18}$ At higher polyion concentrations, where the polyion chains interact with each other, the fast mode is a counterion-accelerated blob diffusion ${ }^{19}$ or a simple gel mode, ${ }^{17}$ respectively.

The slow mode, on the other hand, is not understand despite years of study. Experimentally, dynamic light scattering is the only known means of unambiguously detecting the diffusion mode splitting. Conductivity, ${ }^{20}$ intrinsic viscosity, ${ }^{21}$ fluorescence recovery after photobleaching, ${ }^{22}$ calorimetry, ${ }^{22}$ and other methods show fairly regular monotone trends as a function of $c_{\mathrm{S}}$. Electrophoretic diffusion coefficients, ${ }^{23} D_{\text {ELS }}$, do not reveal any anomaly. $D_{\text {ELS }}$ decreases at low $c_{\mathrm{S}}$ but is always 
larger than $D_{\text {slow }}$. Schmitz and Ramsay ${ }^{24}$ performed dynamic light scattering measurements in the presence of a sinusoidal electric field on poly(lysine) (PLL). They proposed that temporal aggregates controlled by counterion interactions are responsible for the slow mode. However, the slow mode can be observed only at low field strengths. This suggests, that the species or interactions responsible for the slow mode are rather weak and unstable.

There have been proposed further explanations for the slow mode, but none has proved completely found satisfactory. Drifford and Dalbiez ${ }^{2}$ have found an halfempirical relationship between $c_{\mathrm{S}}$ at the point of splitting and the corresponding polyion concentration $c_{\mathrm{P}}$. Due to this theory, the splitting of $D$ into $D_{\text {fast }}$ and $D_{\text {slow }}$ will occur when the counterion concentration contributed by the polyion exceeds the concentration of the added salt.

Often also short-range translation ordering of polyelectrolytes are proposed to the reason for the slow mode. X-Ray scattering ${ }^{25}$ studies on DNA show that the intermolecular spacing decreases with increasing DNA concentration and the local ordering disappears when salt is added. Similar measurements on polylysine, ${ }^{26}$ polyacrylate, ${ }^{27}$ and $\mathrm{t}-\mathrm{RNA}^{8}$ show that the position of the Bragg peak is affected by polymer molar mass, polymer concentration, and salt concentration. Ordering is also predicted by NMR relaxation studies ${ }^{28}$ on NaPSS, suggesting ordering of local domains of $1000 \mathrm{~nm}$ and more. Unfortunately, these studies were carried out at high polyion concentrations, so that there is no evidence that local ordering is relevant at lower ionic strength, where the diffusion mode splitting is observed.

Some people discuss also a dilute-semidilute transition corresponding to a coil-rod transition, but it has been argued to be highly unlikely. ${ }^{29}$

Other theories ${ }^{3,13,16}$ explain the slow mode by the formation of multichain domains, i.e., by clusters. Apparent dimensions of these domains were estimated of the order of $300 \mathrm{~nm}$ and more, irrespective of polyion molar mass. The reason, why these clusters should be formed, are unclear. From the experimental results it can concluded only that the mechanism of cluster formation is of electrostatic nature and that the interaction to give rise to domains is attractive.

Recently, it was reported that the slow mode is not measurable on solutions of heparin, ${ }^{30}$ chondroitin 6sulfate ${ }^{30}$ or acrylamide/sodium acrylate copolymers ${ }^{31}$ when the samples were filtered through a 0.1 or $0.05 \mu \mathrm{m}$ pore-size membrane instead of filtration through conventional $0.2 \mu \mathrm{m}$ membranes. This effect may be interpreted such that the origin of the slow mode resides in very small mass fractions of aggregates, entanglements of polyelectrolytes, or very small particulate contaminants, which are always removable by filtration if the size of the filter is sufficiently small. Other groups ${ }^{7,32}$ report, on the other hand, a gradual reappearance of the filtrated slow mode after a time scale of days, weeks, or months. For instance, Sedlak ${ }^{33}$ has shown that the amplitude and the value of the slow diffusion coefficient become changed by filtration. The apparent radii of the slow mode domains are larger than the diameters of the filter pores, so that the slow mode domains are forced to go through pores smaller than their own dimensions.
This has a weak disrupting effect on the slow-mode domains. $D_{\text {slow }}$ becomes somewhat higher, but it is not possible to remove the slow mode completely. Thus, the slow mode is real, but its physical meaning remains unclear.

In this paper, we report dynamic light scattering studies on poly(diallyl- $N, N$-dimethylammonium chloride) (PDADMAC, $M_{w}=2.5 \times 10^{5} \mathrm{~g} \mathrm{~mol}^{-1}$ ) in aqueous/alcoholic $\mathrm{NaCl}$ and $\mathrm{Na}_{2} \mathrm{SO}_{4}$ salt solutions at various given polyion concentrations. Our aims are threefold: (1) Measurements on PDADMAC are rare and up to yet the fast/slow diffusion splitting phenomenon was not studied with respect to aqueous/alcoholic solvents and to the influence of different salts. The only study, as far as we know, is that of Xia et al. ${ }^{34}$ They have performed dynamic and electrophoretic light scattering measurements as a function of polyion mass in salt free solutions. The results are quite interesting. It could be shown that $D_{\text {slow }}$ depends on $M_{w}$ while $D_{\text {fast }}$ does not. This confirms the thesis that the fast mode is a blob diffusion whereas the slow mode is a multichain diffusion. (2) The detection of internal polymer diffusive modes depends sensitively on the technical equipment used. Fortunately, we have two instruments, an older ALV-3000 correlator and the new ALV-5000 correlator where the latter is equipped with the multiple tau technique. Thus, it is instructive to perform the measurements with both instruments and compare the results. The differences found are enormous and amazing. Both analysis methods predict a slow and a fast mode, but the amplitudes are significantly different and in addition the modern multiple tau technique yields a third diffusion mode which has been observed never before. As a result we present a new model and we will see that there is no fast/slow mode transition at all. (3) The amplitude and the absolute value of a diffusion coefficient depends sensitively on the solvent quality. To study this influence we have changed the kind of the solvent, the polyion concentration, the valence of the counterions, and the ionic strength. We will see that these parameters influence $D_{\text {fast }}$ and $D_{\text {slow }}$ in different ways. Quite interesting are also our results on filtered and unfiltered PDADMAC solutions. We will see that the slow mode is absolutely real.

\section{THEORETICAL FOUNDATIONS}

The most important theory for the dynamics of polyelectrolyte solutions with added salt is the coupled mode theory ${ }^{1,35}$ developed by Schurr and coworkers. However, it is limited to the case where the counterions and coions of the added salt are monovalent and have the same mobility. A generalization to include higher valence and differing mobility is the calculation performed by Tivant et al. ${ }^{18}$ This theory yields an expression for the apparent diffusion coefficient, $D_{\text {app }, z}$, that is measured by dynamic light scattering. It holds:

$$
D_{\mathrm{app}, z}=\frac{1}{2}\left[D_{1}\left(1-t_{1}\right)+D_{2}\left(1-t_{2}\right)+D_{3}\left(1-t_{3}\right)-\sqrt{\Delta}\right]
$$

with 


$$
\begin{aligned}
\Delta= & D_{1}^{2}\left(1-t_{1}\right)^{2}+D_{2}^{2}\left(1-t_{2}\right)^{2}+D_{3}^{2}\left(1-t_{3}\right)^{2} \\
& +2\left[D_{1} D_{2}\left(t_{1} t_{2}-t_{3}\right)+D_{2} D_{3}\left(t_{2} t_{3}-t_{1}\right)\right. \\
& \left.+D_{1} D_{3}\left(t_{1} t_{3}-t_{2}\right)\right]
\end{aligned}
$$

where the subscripts $1-3$ refer to polyion, counterion, and coion, respectively. The dimensionless entities, $t_{i}$, are the transport coefficients. We have

$$
t_{i}=\kappa_{i}^{2} D_{i} / \sum_{i} \kappa_{i}^{2} D_{i}
$$

where

$$
\kappa_{i}^{2}=\frac{e^{2}}{\varepsilon \varepsilon_{0} k_{\mathrm{B}} T} z_{i}^{2} c_{i}
$$

are the partial contributions of the three species to the Debye-Hückel factor $\kappa^{2}$ ( $e$ is the elementary charge, $\varepsilon_{0}$ the influence constant, $\varepsilon$ the dielectric constant of the solvent, $k_{\mathrm{B}}$ the Boltzmann constant, and $T$ the absolute temperature).

For univalent salts and identical counterion and coion diffusion coefficients eq 1 reduces to

$$
D_{\text {app }, z}=\frac{1}{2}\left[D_{\mathrm{P}}(1-\Omega)+D_{\mathrm{S}}(1+\Omega)\right]
$$

where $D_{\mathrm{S}}=D_{2}=D_{3}$ is the average diffusion coefficient for the small ions and $D_{\mathrm{P}}=D_{1}$ the higher salt infinite dilution value of the diffusion coefficient for the polyion. For $\Omega$ it holds

$$
\Omega=\frac{D_{\mathrm{P}}-D_{\mathrm{S}}\left[1+\left(2 c_{\mathrm{S}} / c_{\mathrm{P}}\right) Z_{\text {eff }}^{-1}\right] Z_{\text {eff }}^{-1}}{D_{\mathrm{P}}+D_{\mathrm{S}}\left[1+\left(2 c_{\mathrm{S}} / c_{\mathrm{P}}\right) Z_{\text {eff }}^{-1}\right] Z_{\text {eff }}^{-1}}
$$

where $Z_{\text {eff }}$ is the effective number of elementary charges on the polyion that are not neutralized by the counterions and $c_{\mathrm{P}}$ and $c_{\mathrm{S}}$ are the concentrations of polyion and added salt, respectively. In the dual limits $c_{\mathrm{S}} \gg Z_{\text {eff }} c_{\mathrm{P}}$ and $D_{\mathrm{S}} \gg D_{\mathrm{P}}$, eq 5 reduces to the familiar Donnan equilibrium form

$$
D_{\text {app }}=D_{\mathrm{P}}\left[1+\left(Z_{\text {eff }}^{2} c_{\mathbf{P}} / 2 c_{\mathrm{S}}\right)\right]
$$

As long as there is no slow mode $D_{\text {app }}$ is called the fast or ordinary diffusion coefficient. If the solution is dilute it describes the electrostatic coupling between a polyion and its counterions while if the solution is semidilute $D_{\text {app }}$ refers to the coupling between the small ions and a polyion blob.

The other theory which will be tested here, is the relationship proposed by Drifford and Dalbiez ${ }^{2}$ to calculate the position where the fast/slow diffusion mode splitting happens. It holds:

$$
\frac{c_{\mathrm{P}}^{*}}{\sum_{i} z_{i}^{2} c_{i}^{*}}\left(\frac{1}{\xi}\right)=z_{2}
$$

where $z_{2}$ is the valence of the counterions, $\xi=e^{2} b /$ $\left(4 \pi \varepsilon_{0} \varepsilon k_{\mathrm{B}} T\right)$ the linear charge density parameter of the polyion (with $b$ the average contour distance between two successive charges on the chain), and $c_{\mathrm{P}}^{*}$ and $c_{i}^{*}$ the monomer molar concentrations of the polyion and added salt at the position where the splitting takes place.

The salts used here are $\mathrm{NaCl}$ and $\mathrm{Na}_{2} \mathrm{SO}_{4}$, respectively.
That is, for $\mathrm{NaCl}$ we have

$$
\frac{c_{\mathrm{P}}^{*}}{2 c_{\mathrm{S}}^{*}}\left(\frac{1}{\xi}\right)=1
$$

and for $\mathrm{Na}_{2} \mathrm{SO}_{4}$

$$
\frac{c_{\mathrm{P}}^{*}}{6 c_{\mathrm{S}}^{*}}\left(\frac{1}{\xi}\right)=2
$$

The experiments will be performed at constant $c_{\mathrm{P}}$ where as $c_{\mathrm{S}}$ will be varied. Consequently, the diffusion splitting should happens at $c_{\mathrm{S}}^{*}=c_{\mathrm{P}} / 2 \xi$ for $\mathrm{NaCl}$ and at $c_{\mathrm{S}}^{*}=c_{\mathrm{P}} / 12 \xi$ for $\mathrm{Na}_{2} \mathrm{SO}_{4}$.

\section{MATERIALS AND METHODS}

Poly(diallyl- $N, N$-dimethylammonium chloride) (PDADMAC) was obtained from Polysciences. It was carefully characterized by static and dynamic light scattering at high $\mathrm{NaCl}$-concentrations. The molar mass was found to be $M_{w}=2.5 \times 10^{5} \mathrm{~g} \mathrm{~mol}^{-1}$ in agreement with manufactor's specifications. This corresponds to a degree of polymerization of $P_{w}=1548$. The average distance between the amino-groups is $a=500 \mathrm{pm}$ with respect to the full extended polyion chain.

The water used was deionized, distilled, and filtered through a $0.05 \mu \mathrm{m}$ pore filter. Its conductivity was less than $1 \mu \mathrm{s}$. The alcohol's, methanol, ethanol, and $n$ propanol, were AR grade and purchased from Aldrich. They were mixed in various proportions with water. After that the mixtures were filtered through a special filter for organic solvents (Schleicher and Schuell, pore size $0.2 \mu \mathrm{m}$ ). Thereby, addition of known amount of AR grade salt $\left(\mathrm{NaCl}\right.$ and $\mathrm{Na}_{2} \mathrm{SO}_{4}$ from Merck) provides solvents of the designated salt molarities. Samples of PDADMAC were diluted, dissolved, and vigorously dialyzed for a week against the aqueous/alcoholic salt solvents at $25^{\circ} \mathrm{C}$ with $4-5$ changes of dialysate. The spectra-pore tubes purchased from Spectrum-Medical Industries had a 12000-15000 molar mass cutoff. Finally, the dialyzed PDADMAC-solutions were centrifuged with a Laborfuge (Heraeus) at $11000 \mathrm{~min}^{-1}$ for $2 \mathrm{~h}$ and filled directly into the light scattering cells. Losses in polymer concentration were determined with a conductivity-meter PW-9501 (Philipis) before and after centrifugation.

\section{Dynamic Light Scattering}

Dynamic light scattering measurements were performed with a spectrometer consisting of an $\mathrm{Al}-\mathrm{Sp} 81$ goniometer and two correlators, the old ALV-3000 correlator and the new ALV-5000 multiple tau digital correlator. The light source was a He-Ne laser (Spectra Physics) operating at $632.8 \mathrm{~nm}$ wavelength and $36 \mathrm{~mW}$ power. Calibration was proved by the volume-correlated scattering intensities of toluene in the angular range from $30^{\circ}$ to $150^{\circ}$. It was less than $\pm 1 \%$.

The measurements yield the homodyne intensity-intensity correlation function, $g_{2}(q, t)$, with $q$ the amplitude of the wavevector and $t$ the delay time. For a Gaussian distribution of the intensity profile $g_{2}(q, t)$ can be recalculated into the electric field autocorrelation function, ${ }^{36} g_{1}(q, t)$, by 


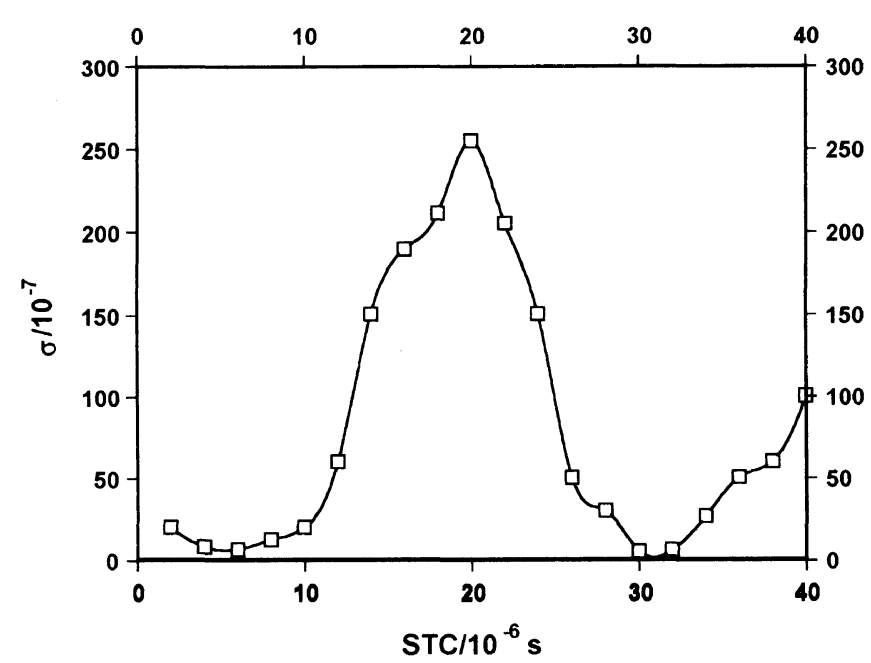

Figure 1. Sample time clock, $\Delta t$, dependence of the standard deviation, $\sigma$, for PDADMAC dissolved in $25 \mathrm{~mol} \%$ methanol and $75 \mathrm{~mol} \%$ water at $c_{\mathbf{P}}=0.05 \mathrm{M}, c_{\mathrm{S}}=0.1 \mathrm{M} \mathrm{NaCl}$, and $\theta=90^{\circ}$.

$$
g_{2}(q, t)=B\left(1+\beta\left|g_{1}(q, t)\right|^{2}\right)
$$

where $B$ is the baseline and $\beta$ a constant that takes into account the number of coherence areas that generates the signal $(0<\beta<1)$. When $q\left\langle R^{2}\right\rangle_{z}^{1 / 2} \ll 1$, and when the sample is monodisperse, $g_{1}(q, t)$ is a single exponential function

$$
g_{1}(q, t)=\exp (-t / \tau)=\exp (-\Gamma t)=\exp \left(-q^{2} D t\right)
$$

where $\tau$ is the relaxation time, $\Gamma=1 / \tau$ the relaxation rate, and $D=\lim _{q \rightarrow 0}\left(\Gamma / q^{2}\right)$ the translational diffusion coefficient.

Unfortunately, nearly all samples are polydisperse so that $g_{1}(q, t)$ must be expressed as an integral of the exponential decays weighted over the distribution, $A(\tau)$, of the relaxation times

$$
g_{1}(t)=\int_{0}^{\infty} A(\tau) \exp (-t / \tau) \mathrm{d} \tau
$$

This Laplace-integral can be analyzed by different methods. Here, we use the cumulant method ${ }^{37}$ and the CONTIN 2DP program. ${ }^{38,39}$ The latter yields directly $A(\tau)$ by inverse Laplace transformation, employing the constrained regularization method.

With the old ALV-3000 correlator different sample times, $\Delta t$, were used to check the consistence of the diffusion coefficient values. The diffusion modes were separated by measuring the fast mode at short sample time clocks (typically $6 \mu \mathrm{s}$ ) and the slow mode at long times (typically $30 \mu \mathrm{s}$ ). The optimal range of sample time clocks is determined by recording the correlation functions at $\theta=90^{\circ}$ varying $\Delta t$ and then judging the quality of the fit from residual plots and $\sqrt{\sigma^{2}}$ values. A typical plot of $\sqrt{\sigma^{2}}$ versus $\Delta t$ is shown in Figure 1 . We observe two minima at $\Delta t \approx 6 \mu \mathrm{s}$ and $\Delta t \approx 30 \mu \mathrm{s}$. The sample time clocks for the other angles were determined by keeping the product $q^{2} \Delta t=$ const. Such an optimization of $\Delta t$ is very important. If $\Delta t$ is correct, $D_{\mathrm{app}, z}$ versus $q^{2}$ gives a straight line (see Figure 2a), whereas if $\Delta t$ is incorrect $D_{\text {app }, z}$ scatters strongly (see Figure $2 \mathrm{~b}$ ).

In contradiction to this, the work with the new multiple tau ALV-5000 digital correlator is much easier and much
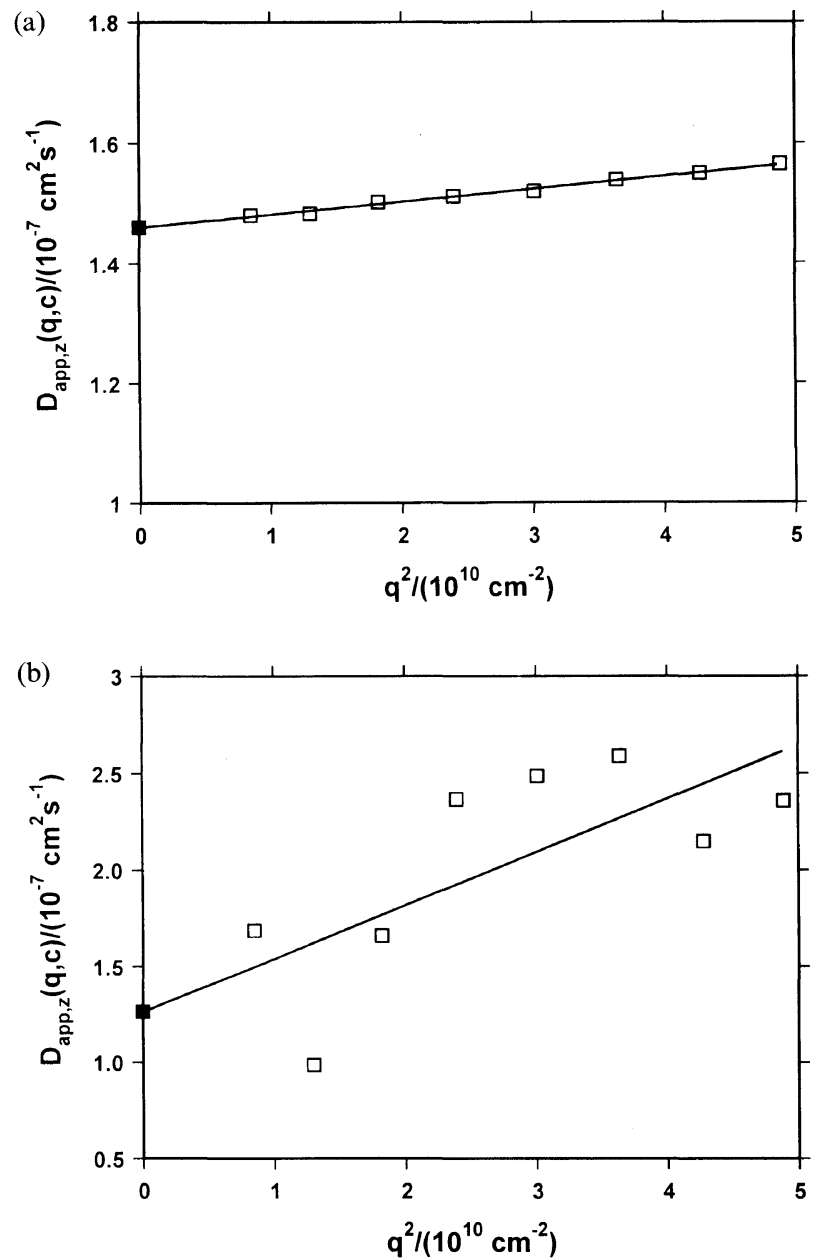

Figure 2. Typical plots of $D_{\mathrm{app}, z}$ versus $q^{2}$ for PDADMAC dissolved in $25 \mathrm{~mol} \%$ methanol and $75 \mathrm{~mol} \%$ water at $c_{\mathrm{P}}=0.05 \mathrm{M}$ and $c_{\mathrm{S}}=0.1 \mathrm{M}$ $\mathrm{NaCl}$. (a) $\Delta t=6 \mu \mathrm{s}$; (b) $\Delta t=18 \mu \mathrm{s}$.

less time consuming. There is no need of adjusting the sampling time at all and nevertheless the performance is always best. There will be built blocks of 8 channels with constant sampling time and the sampling time is doubled from one block to another. For example, with 320 correlation channels, lag times from $12.5 \mathrm{~ns}$ to many hours can be processed in parallel, with 39 different sampling times used.

\section{RESULTS AND DISCUSSION}

First, we discuss the results obtained with the more conventional method, using the older ALV-3000 correlator. We do then inspect the results derived with the modern multiple tau technique and finally we make a comparison.

\section{Results Obtained with the Conventional Method}

Figure 3 shows the apparent diffusion coefficient, $D_{\text {app }, z}$, extrapolated to $q=0$ versus $c_{\mathrm{NaCl}}$ for PDADMAC at $8 \mathrm{~g}^{-1}$ and $T=25^{\circ} \mathrm{C}$. We see that $D_{\mathrm{app}, z}$ first rises with decreasing salt concentration and then drops and decreases. That is, we have a behavior which is typical for a fast/slow diffusion mode splitting. The vertical line presents the position where the splitting should appear theoretically and indeed this mark $\left(c_{\mathrm{S} \text {,theory }}^{*}=\right.$ $1.3 \times 10^{-2} \mathrm{M}$ ) agrees quite well with that observed ex- 


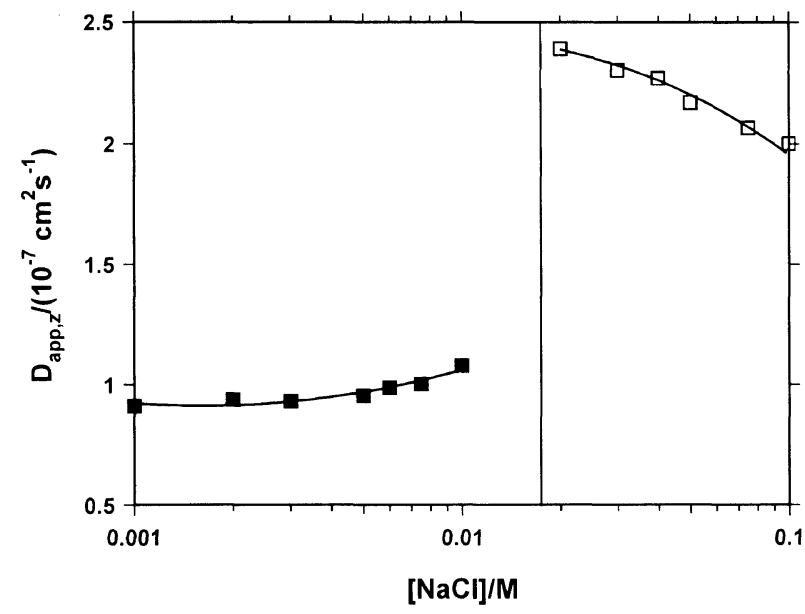

Figure 3. $D_{\mathrm{app}, z}$ extrapolated to zero scattering angle $\left(q^{2}=0\right)$ as a function of $c_{\mathrm{NaCl}}$ at $c_{\mathrm{P}}=8 \mathrm{~g}^{-1}$ and $T=25^{\circ} \mathrm{C}$ for pure water. ( $\square$ ) fast mode; ( $)$ slow mode. The vertial line presents the position of the diffusion mode splitting predicted by the Drifford-Dalbiez formula (eq 8). The above line is the best fit calculated by the coupled-ion theory (eq 5).

perimentally. For $c_{\mathrm{S}}>c_{\mathrm{S}}^{*}$ there is only one diffusion mode, the fast mode. It can be well described by the coupled mode theory, i.e., by eq 5 . With $D_{\mathrm{S}}=16.8 \times 10^{-6} \mathrm{~cm}^{2} \mathrm{~s}^{-1}$ and $D_{\mathrm{P}}=1.96 \times 10^{-8} \mathrm{~cm}^{2} \mathrm{~s}^{-1}$ at $c_{\mathrm{S}}=1 \mathrm{M}$ a least square fit gives $Z_{\text {eff }}=12.1$. For $c_{\mathrm{S}}<c_{\mathrm{S}}^{*}$ we observe only a slow mode where $D_{\text {slow }}$ increases slightly with increasing $c_{\mathbf{S}}$. This bizarre behavior is the same as that observed for NaPSS, ${ }^{19}$ which is an anionic polyion.

Figures 4 and 5 show plots of $D_{\mathrm{app}, z}$ versus $c_{\mathrm{NaCl}}$ at $c_{\mathrm{P}}=$ $6 \mathrm{gl}^{-1}$ and $c_{\mathrm{P}}=8 \mathrm{gl}^{-1}$, respectively, where the solvent now is a mixture of $25 \%$ molar fraction methanol and $75 \%$ water at $T=25^{\circ} \mathrm{C}$. The mode splitting takes place again near the theoretical mark of $c_{\mathrm{NaCl}, \text { theory }}=1 \times 10^{-2} \mathrm{M}$. This mark is somewhat lower than that for poor water, that is $c_{\mathrm{S}}^{*}$ decreases with increasing methanol content as predicted by theory. ${ }^{3}$ However, this behavior depends on $c_{\mathrm{P}}$. At $c_{\mathrm{P}}=6 \mathrm{gl}^{-1}$ there is only a slow mode whereas at $c_{\mathrm{P}}=8 \mathrm{~g}^{-1}$ and $c_{\mathrm{S}}<c_{\mathrm{S}}^{*}$ we obtain both a slow and a fast diffusion mode. Such a behavior is not unusual. It is also found for NaPSS and DNA, ${ }^{3,4}$ respectively. For these polyions at moderate polyion molar masses both modes are present simultaneously, while at high molar masses the slow mode is the only mode.

For $c_{\mathrm{P}}=8 \mathrm{~g}^{-1}$ we have enough $D_{\text {app }}$-values to calculate the effective polyion charge, $Z_{\text {eff }}$, using eq 5 . With $D_{\mathrm{S}}=9.12 \times 10^{-6} \mathrm{~cm}^{2} \mathrm{~s}^{-1}$ and $D_{\mathrm{P}}=3.0 \times 10^{-8} \mathrm{~cm}^{2} \mathrm{~s}^{-1}$, where the last value was obtained by extrapolation from $D_{\text {fast }}$ to $c_{\mathrm{s}}=1 \mathrm{M}$, it follows $Z_{\text {eff }}=9.2$. This value is lower than the value $Z_{\text {eff }}=12.1$ of pure water. According to counterion condensation theory ${ }^{40}$ for univalent counterions the polyion charge $Z$ which it not screened by counterions is $Z=P / \xi$ where $P$ is the degree of polyion polymerization and $\xi$ the charge density parameter. Here, for $P=1548$ and water $Z$ is 1086 while for $25 \%$ methanol $/ 75 \%$ water we have $Z=840$. These $Z$-values are clearly too high, but they show the same dependence on the solvent composition as $Z_{\text {eff }}$. This effect is not unusual. It has also been observed in electrophoretic studies $^{41}$ on DNA. The simplest reason is that $Z$ refers to the entire polyion while $Z_{\text {eff }}$ refers to a polyion blob. Assuming that the number of segments of a blob can be

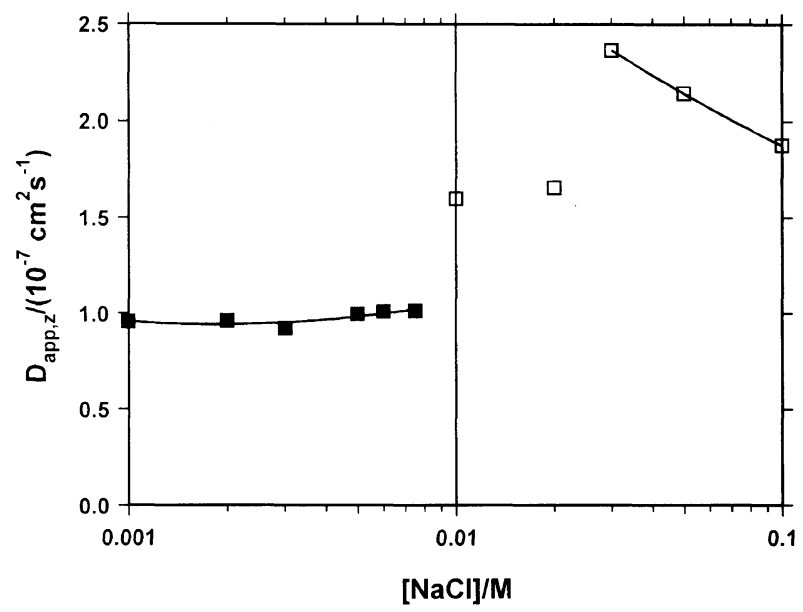

Figure 4. $D_{\mathrm{app}, \mathrm{z}}$ extrapolated to $q^{2}=0$ as a function of $c_{\mathrm{NaCl}}$ at $c_{\mathrm{P}}=$ $6 \mathrm{~g} \mathrm{l}^{-1}$ and $T=25^{\circ} \mathrm{C}$ for a mixture of $25 \mathrm{~mol} \%$ methanol and $75 \mathrm{~mol} \%$ water. The vertical line presents the position of the diffusion mode splitting predicted by the Drifford-Dalbiez formula (eq 8). Symbols as in Figure 3.

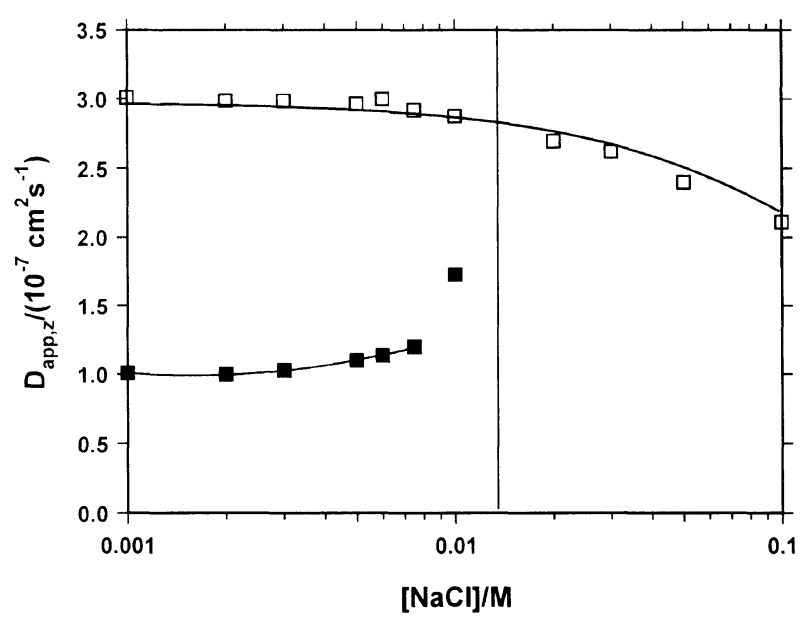

Figure 5. $D_{\mathrm{app}, z}$ extrapolated to $q^{2}=0$ as a function of $c_{\mathrm{NaCl}}$ at $c_{\mathrm{P}}=$ $8 \mathrm{gl}^{-1}$ and $T=25^{\circ} \mathrm{C}$ for a mixture of $25 \mathrm{~mol} \%$ methanol and $75 \mathrm{~mol} \%$ water. Symbols as in Figure 3.

calculated as $g_{\mathrm{b}}=Z_{\text {eff }} \cdot \xi$, we obtain $g_{\mathrm{b} \text {,water }}=17.2$ and $g_{\mathrm{b} \text {,water,methanol }}=16.9$; that is $g_{\mathrm{b}}$ seems to be independent on the solvent quality.

The most interesting curves may be that presented in Figures 6 and 7. In Figure $6, D_{\text {app }, z}$ is plotted versus $c_{\mathrm{NaCl}}$ for $c_{\mathrm{p}}=8 \mathrm{~g} \mathrm{l}^{-1}, T=25^{\circ} \mathrm{C}$, and $x_{\text {methanol }}=50 \%$. The result is only a single mode, the fast mode. Biexponential fitting gives no reproducible results, and there is nearly no dependence of $D_{\text {app, } z}$ on the sample time clock. This is also the case for the system PDADMAC $/ 25 \%$ methanol $/ 75 \%$ water, $c_{\mathrm{P}}=8 \mathrm{gl}^{-1}, T=25^{\circ} \mathrm{C}$ when $\mathrm{NaCl}$ is exchanged by $\mathrm{Na}_{2} \mathrm{SO}_{4}$ (see Figure 7). Thus, we can conclude that the occurrence of the diffusive mode splitting depends on both the molar fraction of the organic additive as well as on the valence of the counterions used. The higher the alcoholic content of the solvent mixture and the higher the valence of the counterions the higher is the probability that there is no fast/slow mode splitting but only one single mode, the fast mode.

We have performed further measurements on water/ ethanol mixtures. The results are similar to those of water/methanol. A splitting is observed near the theoretical mark proposed by Drifford and Dalbiez ${ }^{2}$ where the 


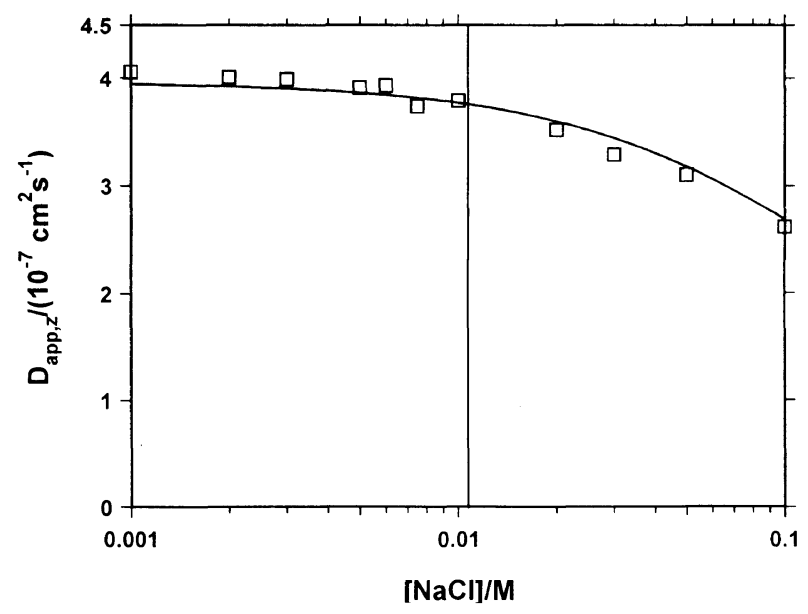

Figure 6. $D_{\mathrm{app}, z}$ extrapolated to $q^{2}=0$ as a function of $c_{\mathrm{NaCl}}$ at $c_{\mathrm{P}}=$ $8 \mathrm{gl}^{-1}$ and $T=25^{\circ} \mathrm{C}$ for a mixture of $50 \mathrm{~mol} \%$ methanol and $50 \mathrm{~mol} \%$ water. Symbols as in Figure 3.

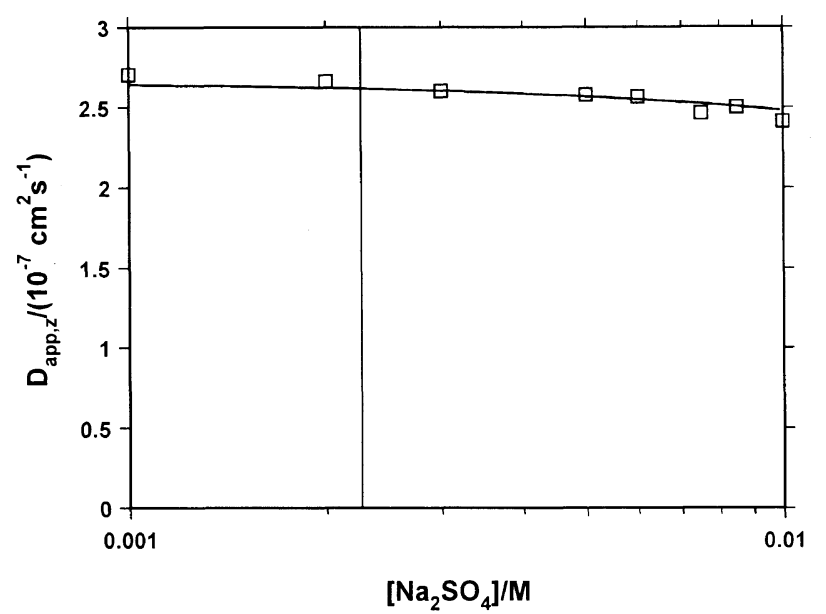

Figure 7. $D_{\text {app }, z}$ extrapolated to $q^{2}=0$ as a function of $c_{\mathrm{Na}_{2} \mathrm{SO}_{4}}$ at $c_{\mathrm{P}}=$ $8 \mathrm{~g} \mathrm{l}^{-1}$ and $T=25^{\circ} \mathrm{C}$ for a mixture of $25 \mathrm{~mol} \%$ methanol and $75 \mathrm{~mol} \%$ water. Symbols as in Figure 3.

fast mode curve can be well fitted through eq 5 . For $25 \%$ ethanol $/ 75 \%$ water and $8 \mathrm{gl}^{-1}$ of PDADMAC $Z_{\text {eff }}$ is 8 , a value which is nearly as large as that for water/ methanol. The absolute values of $D_{\text {fast }}$ and $D_{\text {slow }}$ are, on the other hand, somewhat larger than for water/ methanol. This could be expected, because the viscosity of pure ethanol is larger than that of pure methanol. As a consequence the ratio $\left(D_{\text {fast }} / D_{\text {slow }}\right)_{\text {water/ethanol }} /\left(D_{\text {fast }} /\right.$ $\left.D_{\text {slow }}\right)_{\text {water/methanol }}$ is nearly as large as the viscosity ratio $\eta_{\text {water/ethanol }} / \eta_{\text {water/methanol }}$.

The dimensions of the fast and slow-mode polyion domains can be approximated by the Einstein-Stokes relation $\xi=k_{\mathrm{B}} T / 6 \pi \eta D_{\mathrm{app}, z}$, where $\xi$ is the correlation length of the domains. For the fast mode we find the ratio $\xi_{\text {water } / \text { ethanol }} / \xi_{\text {water } / \text { methanol }} \approx 1.86$ at $c_{\mathrm{S}}=2 \times 10^{-3} \mathrm{M}$, indicating that coupled polyion/small ion diffusion processes are more dominant the higher the viscosity of the solvent is. For the slow mode, on the other hand, we have $\xi_{\text {slow,water/ethanol }} / \xi_{\text {slow,water/methanol }} \approx 1.06$, that is, there is no change in the slow mode dimensions when ethanol is exchanged by methanol.

Exchanging $\mathrm{NaCl}$ by $\mathrm{Na}_{2} \mathrm{SO}_{4}$ we find that $\left(D_{\text {fast }} /\right.$ $\left.D_{\text {slow }}\right)_{\mathrm{Na}_{2} \mathrm{SO}_{4}}=14.5$ is nearly therefold as large as the ratio $\left(D_{\text {fast }} / D_{\text {slow }}\right)_{\mathrm{NaCl}} \approx 5$. A similar result was found by Ferrari

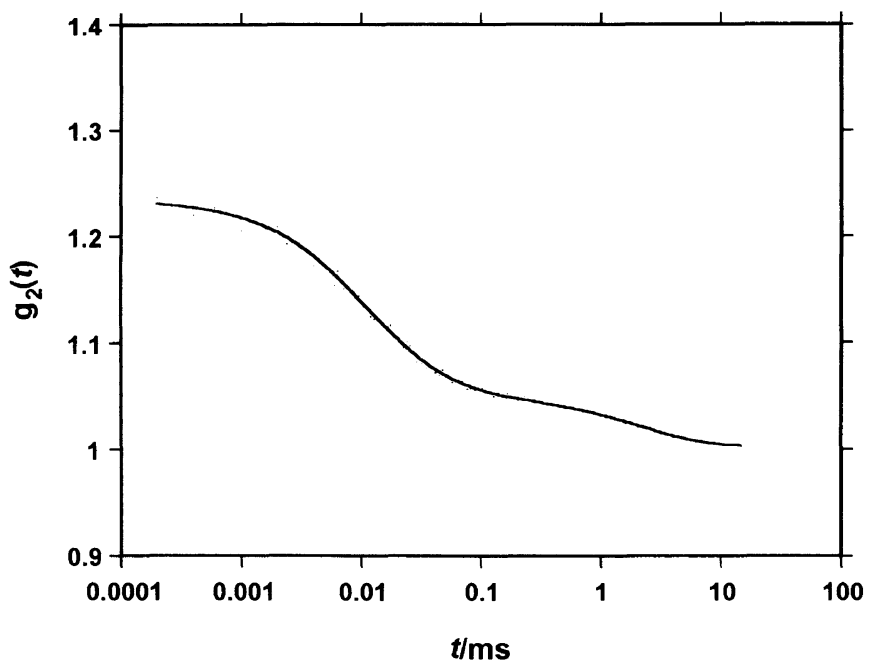

Figure 8. Typical normalized autocorrelation function obtained for PDADMAC dissolved in a mixture of $25 \mathrm{~mol} \%$ methanol and $75 \mathrm{~mol} \%$ water at $c_{\mathrm{P}}=10 \mathrm{gl}^{-1}, c_{\mathrm{NaCl}}=10^{-3} \mathrm{M}$, and $\theta=90^{\circ}$.

and Bloomfield ${ }^{42}$ for mononucleosomal DNA dissolved in pure water. They could show that $D_{\mathrm{fast}, \mathrm{Na}}$ is larger than $D_{\text {fast,Ca }}$ at low salt concentration. Qualitatively, the same trend is observed for PDADMAC, where $D_{\mathrm{fast}, \mathrm{Cl}}$ is larger than $D_{\text {fast, } \mathrm{SO}_{4}}$. Additionally, the effective polyion charge, $Z_{\text {eff }}=3.9$, obtained for $\mathrm{SO}_{4}^{2-}$, where $D_{\mathrm{S}}$ is $8.45 \times 10^{-6}$ $\mathrm{cm}^{2} \mathrm{~s}^{-1}, D_{\mathrm{P}}=3.79 \times 10^{-7} \mathrm{~cm}^{2} \mathrm{~s}^{-1}$ and the solvent $25 \%$ ethanol $/ 75 \%$ water, is nearly exactly half as large that for $\mathrm{Cl}^{-}$.

\section{Results Obtained with the Multiple Tau Technique}

The above given results are in line with the results of other studies and they are also in line with theory. Therefore it may be surprising and amazing that the results which will be presented now are both quantitatively as well as qualitatively different from those.

Figure 8 shows a typical autocorrelation function for PDADMAC obtained by the multiple tau technique. The parameters used are $\theta=90^{\circ}, c_{\mathrm{NaCl}}=10^{-3} \mathrm{M}, c_{\mathrm{P}}=10 \mathrm{~g} \mathrm{l}^{-1}$, $x_{\text {methanol }}=25 \%$, and $T=25^{\circ} \mathrm{C}$. CONTIN 2DP fits this autocorrelation function quite well and gives a bimodal distribution, from which the fast mode is located as $D_{\text {fast }}=9.07 \times 10^{-7} \mathrm{~cm}^{2} \mathrm{~s}^{-1}$ and the slow mode as $D_{\text {slow }}=$ $3.8 \times 10^{-9} \mathrm{~cm}^{2} \mathrm{~s}^{-1}$. For the same system the old conventional analysis method yields $D_{\text {fast }}=3.0 \times 10^{-7} \mathrm{~cm}^{2} \mathrm{~s}^{-1}$ and $D_{\text {slow }}=1.0 \times 10^{-7} \mathrm{~cm}^{2} \mathrm{~s}^{-1}$. That is, two modes are found with both techniques, but the absolute values of the diffusion coefficients are significantly different.

In Figure 9 the angle dependencies of $D_{\text {fast }}$ and $D_{\text {slow }}$ are plotted. Both diffusion coefficients are independent on $\sin ^{2}(\theta / 2)$. The same effect is observed by the conventional method. It may indicate that the particle structures causing the two motions are uniformly in size. The result is, however, somewhat surprising because Xia et $a l .{ }^{34}$ have found an angular dependence for PDADMAC, although only for $D_{\text {slow }}$. The reason for this discrepancy may be the correlator and the experimental conditions used. Xia et al. have performed their measurements in the dilute region in salt free solutions, while our solutions contain salt and the regime is rather semidilute. Additionally, the molar masses used by Xia and us are different. 


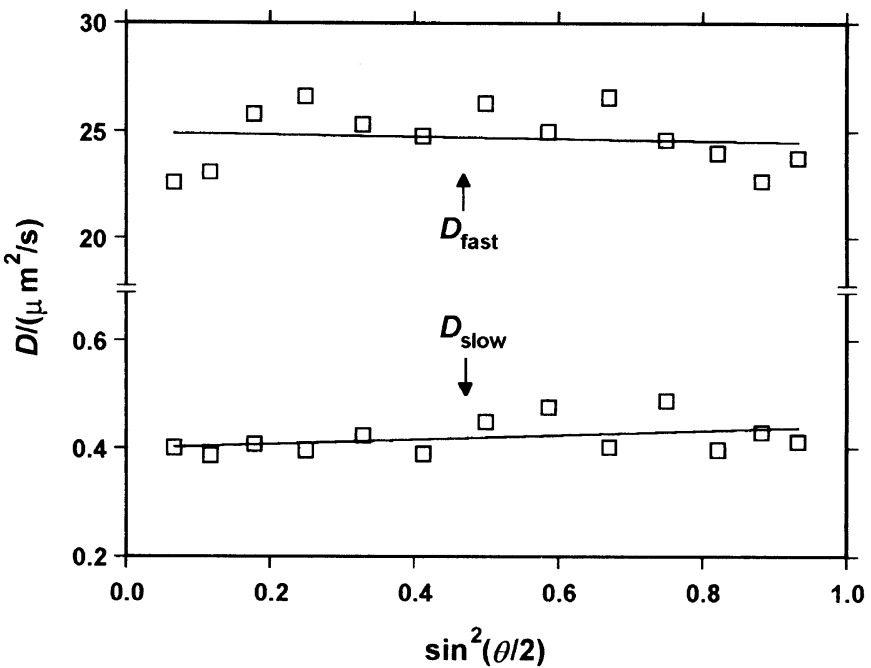

Figure 9. Scattering angle, $\theta$, dependence of the diffusion coefficients, $D_{\text {fast }}$ and $D_{\text {slow }}$, for PDADMAC dissolved in $25 \mathrm{~mol} \%$ ethanol and $75 \mathrm{~mol} \%$ water at $c_{\mathrm{P}}=8 \mathrm{~g} \mathrm{l}^{-1}$ and $c_{\mathrm{Na}_{2} \mathrm{SO}_{4}}=5 \times 10^{-3} \mathrm{M}$.

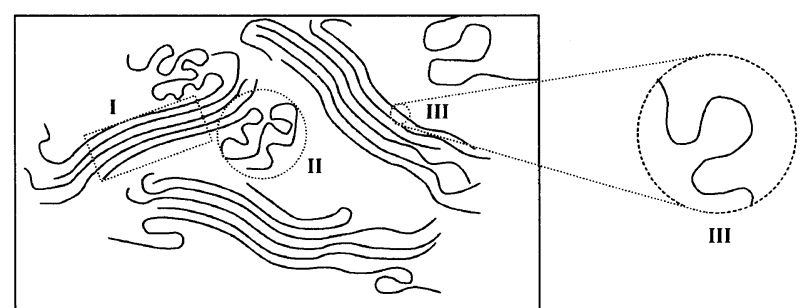

Figure 10. Section of a semidilute polyion solution. Three regions can be distinguished: (I) ordered polyion cluster; (II) polyion heterogeneity regions; (III) polyion blobs.

Before we present the next results it is helpful to introduce a model by which the results, that are somewhat exciting, become understandable. Figure 10 shows a section of the volume of a semidilute polyion solution. We propose that there are three different kinds of regions of nearly identical structure. These are: (1) regions of ordered polyion clusters, (2) regions of hetgerogeneities, and (3) blob regions. A polyion cluster region contains several polyion chains and may have crystalline structure. The order is formed due to electrostatic repulsion between the chains, while the size and stability of a cluster is limited and perturbed by Brownian motion. The average range of the ordering force is the correlation length, $\xi_{\mathrm{s}}$, calculable by the Einstein-Stokes relation. The stronger the electrostatic repulsion between the chains the larger is $\xi_{\mathbf{s}}$. Since the polyion chains are not all equally large and since there are fluctuations in temperature, salt concentration, or polyion concentration, the clusters are not ideal. Parts of the polyion chains hang out of the clusters and build unordered coils. In addition, there are single isolated polyion chains that are not part of any cluster ensemble, but free. We call these coils and partitions of polyion chains heterogeneities. The average dimension, $\xi_{\mathrm{h}}$, of such a unoriented region can be as large or larger than a polyion cluster, but it can be also very small, in extreme as small as a polyion blob. Its size depends very sensible on the polyion type and the conditions of measurement.

The third region describes a polyion blob. This is a region within a single polyion chain. Its average dimen-
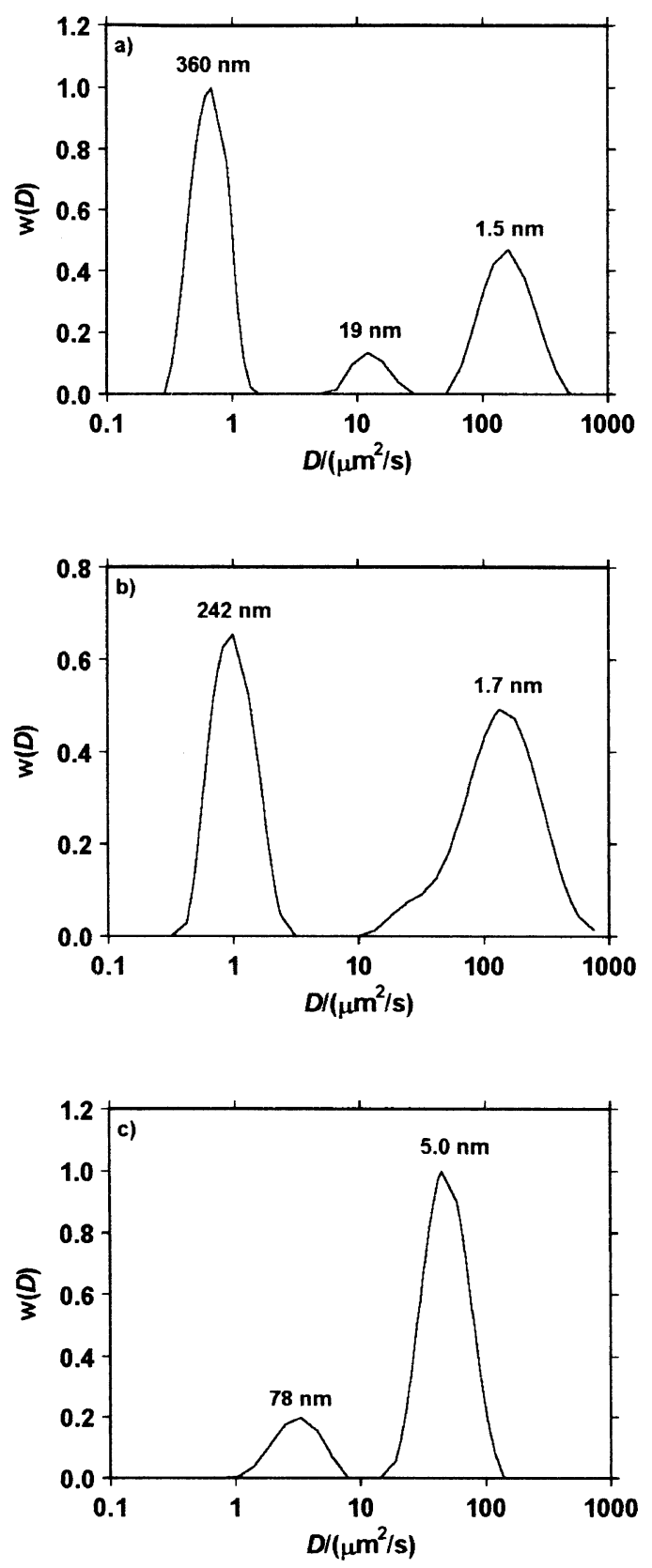

Figure 11. Diffusion coefficient distributions, $w(D)$, for PDADMAC dissolved in pure water at $c_{\mathrm{P}}=8 \mathrm{~g}^{-1}$ where $c_{\mathrm{NaCl}}$ is a) $10^{-3} \mathrm{M}, \mathrm{b}$ ) $10^{-2} \mathrm{M}$, and c) $10^{-1} \mathrm{M}$.

sion is $\xi_{\mathrm{b}}$ and it contains in average $g_{\mathrm{b}}$ polyion segments. $g_{\mathrm{b}}$ is much smaller than the degree of polymerization, $P$, of a polyion and the segments of a blob are coiled in a way that the blob behaves as if it would be in a dilute solution. There are only intramolecular segment/segment interactions and these are screened out over larger distances than $\xi_{\mathrm{b}}$. As a consequence $\xi_{\mathrm{b}}$ is independent on polyion molar mass, but dependent on polyion charge density and solvent quality. If all charges are neutralized, it should be hold $\xi_{\mathrm{b}} \propto c_{\mathrm{p}}^{-v /(3 v-1)}$ where $v$ is 0.5 at theta conditions and 0.6 in a good solvent.

It is not our intention to give a regorous evidence for this model. That is not possible. The model is only a proposal for further discussion. However, if the model is proper, there should be exist theoretically three diffusive modes, one for each structure region. We call them $D_{\text {slow }}, D_{\text {hetero }}$, and $D_{\text {fast }}$, where $D_{\text {slow }}$ stands for the 

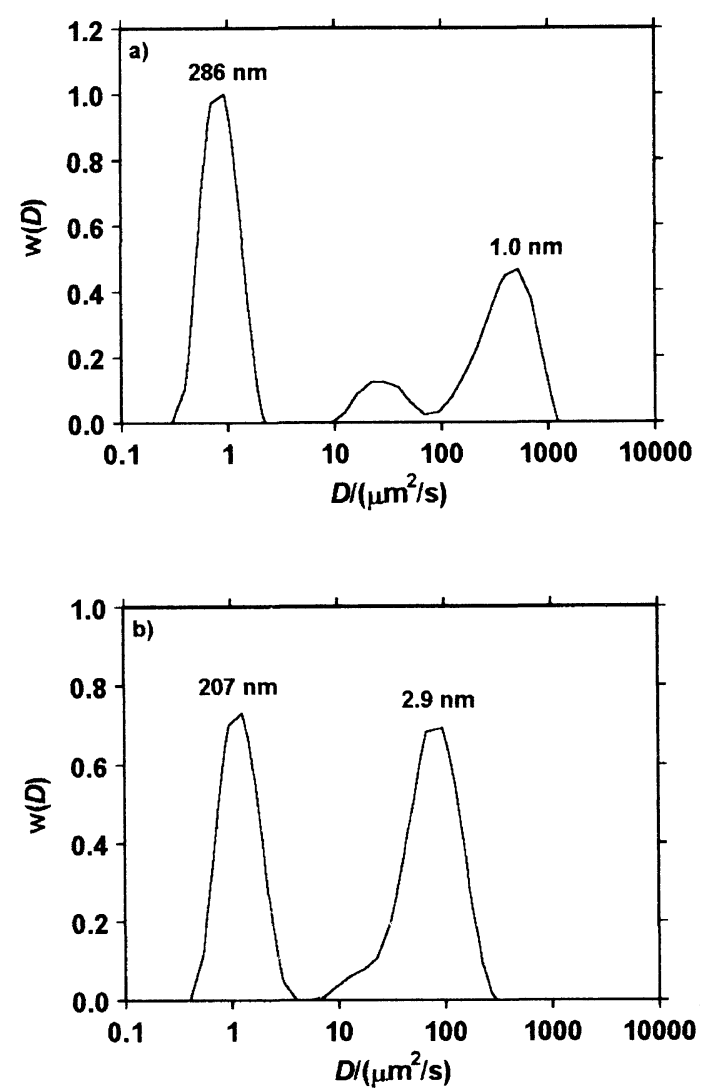

Figure 12. Diffusion coefficient distributions, $w(D)$, for PDADMAC dissolved in pure water at $c_{\mathrm{P}}=8 \mathrm{~g}^{-1}$ where $c_{\mathrm{Na}_{2} \mathrm{SO}_{4}}$ is a) $10^{-3} \mathrm{M}$, and b) $10^{-2} \mathrm{M}$.

cluster regions, $D_{\text {hetero }}$ for the heterogeneities, and $D_{\text {fast }}$ for the blobs. Since $\xi_{\mathrm{s}} \geq \xi_{\mathrm{h}} \geq \xi_{\mathrm{b}}, D_{\text {hetero }}$ may be under certain conditions equal to $D_{\text {slow }}$ or $D_{\text {fast }}$, respectively. The new and interesting question is now: Is there a third diffusive mode or not. With the conventional analysis method only two modes were found.

Figure 11 shows a typical result. It represents the normalized diffusion coefficient distribution, $w(D)$, for the system PDADMAC/water, $c_{\mathrm{P}}=8 \mathrm{gl}^{-1}$, at $T=25^{\circ} \mathrm{C}$ where $c_{\mathrm{NaCl}}$ was varied between $10^{-3}$ and $10^{-1} \mathrm{M}$. There are three peaks indeed, but the number of observable peaks depends on the salt concentration used. For $c_{\mathrm{NaCl}}=10^{-3} \mathrm{M}$ the left peak describes the cluster region motions, the middle peak the heterogeneity diffusion, and the right peak the blob motions. The corresponding correlation lengths calculated by the Einstein-Stokes relation, using the maximum position of the peaks, are $360 \mathrm{~nm}, 19 \mathrm{~nm}$, and $1.5 \mathrm{~nm}$, respectively. When the salt concentration increases to $c_{\mathrm{NaCl}}=10^{-2} \mathrm{M}$, the middle and the right peak run together. Approximately it holds $\xi_{\mathrm{S}}=243 \mathrm{~nm}, \xi_{\mathrm{h}}=10 \mathrm{~nm}$, and $\xi_{\mathrm{b}}=1.7 \mathrm{~nm}$. This means that $\xi_{\mathrm{S}}$ and $\xi_{\mathrm{h}}$ become smaller with increasing $c_{\mathrm{NaCl}}$ while $\xi_{\mathrm{b}}$ becomes larger. At $c_{\mathrm{NaCl}}=10^{-1} \mathrm{M}$ there are only two peaks. The amplitude of the cluster region peak is very small and also $\xi_{\mathrm{S}}$ is diminished to $78 \mathrm{~nm}$. The reason is clear. The polyion charges are screened by the salt ions so that it becomes more and more difficult to build cluster regions which are stabilized by strong enough electrostatic forces. If the salt concentration becomes once more higher this peak vanishes at all. The right peak is the result of the overlapping of the heterogeneity and the 802
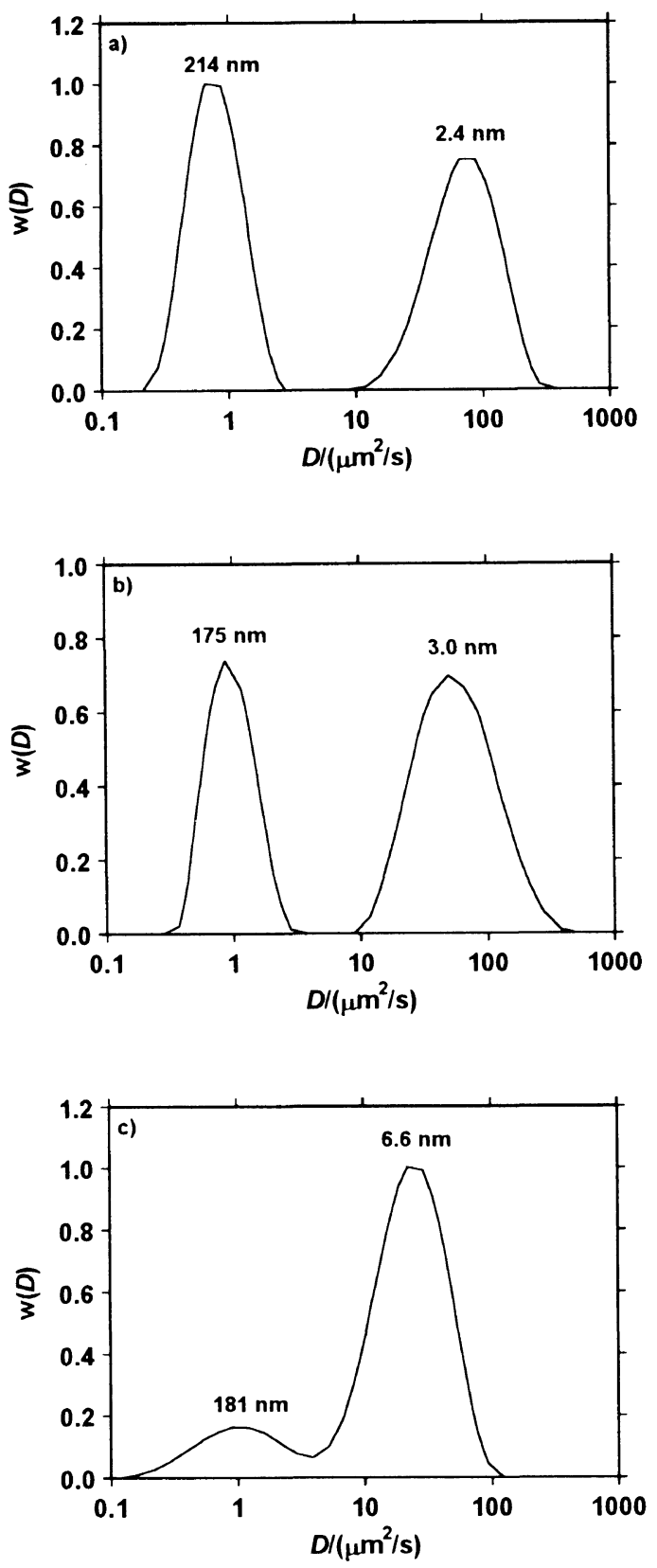

Figure 13. Diffusion coefficient distributions, $w(D)$, for PDADMAC dissolved in a mixture of $25 \mathrm{~mol} \%$ methanol and $75 \mathrm{~mol} \%$ water at $c_{\mathrm{P}}=8 \mathrm{gl}^{-1}$ where $C_{\mathrm{NaCl}}$ is a) $2 \times 10^{-3} \mathrm{M}$, b) $10^{-2} \mathrm{M}$, and c) $10^{-1} \mathrm{M}$.

blob diffusion coefficient distribution, where the contribution of the heterogeneity mode builds the left flank of this peak. For the position of the maximum of this peak we can calculate the correlation length $\xi=w_{\mathrm{b}} \xi_{\mathrm{b}}+$ $\left(1-w_{\mathrm{b}}\right) \xi_{\mathrm{h}}$ where $w_{\mathrm{b}}$ is the weighting factor for the blob mode. Due to the mathematical process how CONTIN 2DP works the maximum position is predominantly influenced by the blob mode so that $w_{\mathrm{b}}$ is $\approx 1$ and $\xi \approx \xi_{\mathrm{b}}$. Its value is $5 \mathrm{~nm}$ which is larger than $1.7 \mathrm{~nm}$ so that the thesis that a blob becomes larger with increasing salt concentration seems to be right.

These effects are not unique. Figure 12 shows the diffusion modes of PDADMAC when the salt $\mathrm{NaCl}$ is exchanged by $\mathrm{Na}_{2} \mathrm{SO}_{4}$. Principally, the same effect is found as for $\mathrm{NaCl}$. At $c_{\mathrm{Na}_{2} \mathrm{SO}_{4}}=10^{-3} \mathrm{M}$ we have three peaks while at $10^{-2} \mathrm{M}$ the middle and the right peak run together. The only difference is that the correlation 

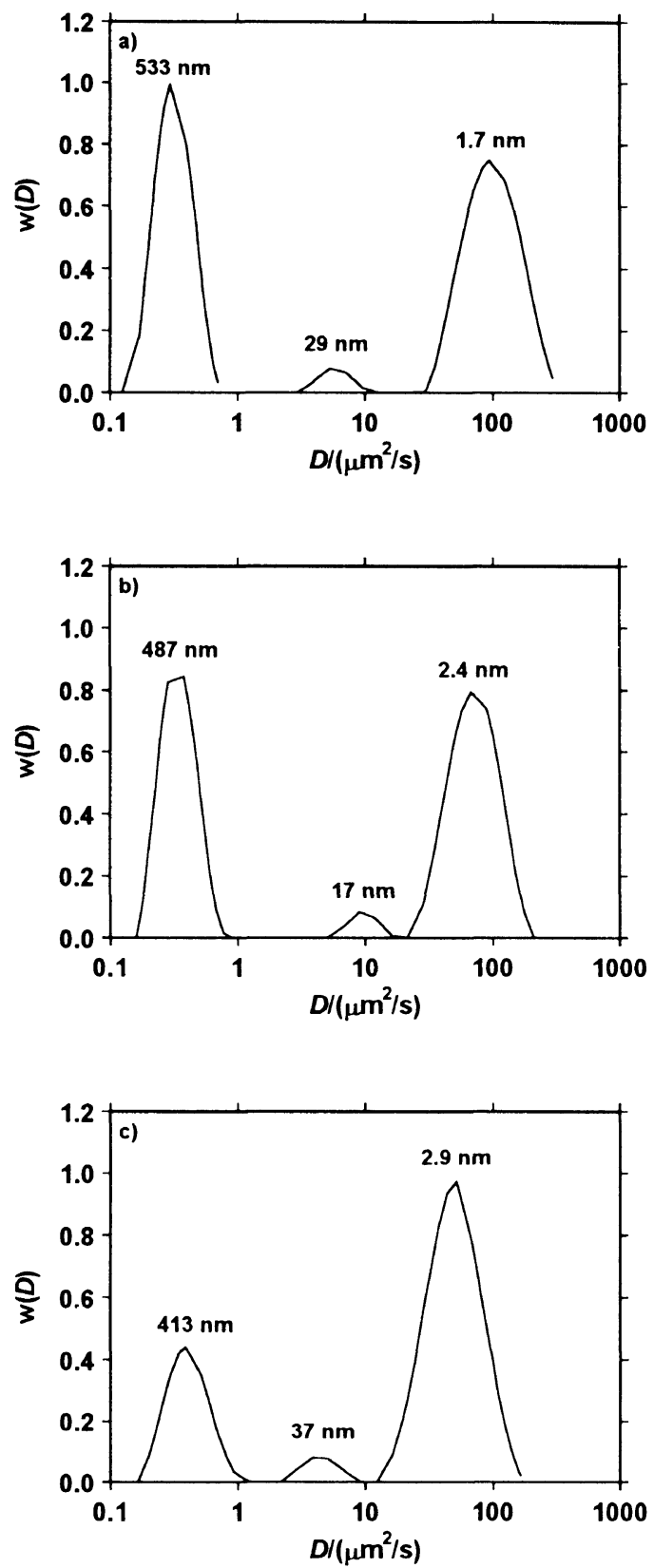

Figure 14. Diffusion coefficient distributions, $w(D)$, for PDADMAC dissolved in a mixture of $25 \mathrm{~mol} \%$ methanol and $75 \mathrm{~mol} \%$ water at $c_{\mathrm{P}}=30 \mathrm{gl}^{-1}$ where $c_{\mathrm{NaCl}}$ is a) $10^{-3} \mathrm{M}$, b) $5 \times 10^{-2} \mathrm{M}$, and c) $10^{-1} \mathrm{M}$.

lengths $\xi_{\mathrm{S}}, \xi_{\mathrm{h}}$, and $\xi_{\mathrm{b}}$ are somewhat smaller than those of $\mathrm{NaCl}$. Since the valence of $\mathrm{SO}_{4}$ is twice that of $\mathrm{Cl}$ this is understandable.

Figure 13 presents the results obtained for the system $x_{\text {water }}=75 \%, x_{\text {methanol }}=25 \%$, and $c_{\mathrm{P}}=8 \mathrm{gl}^{-1}$. Now, there are only two peaks. The cluster peak is quite well isolated, but the heterogeneous and the blob modes run together to build one peak. This may indicate that the dimensions of the heterogeneity regions are rather small, in especially smaller than in pure water. This is understandable since the solvent quality is more poorer the higher the alcoholic content is. The influence of the salt concentration is, on the other hand, the same as before. The amplitude and the correlation length of the cluster peak decreases with increasing salt concentration and for $c_{\mathrm{NaCl}}>0.1 \mathrm{M}$ all three peaks run together. However, this holds not at general. The quality of the decomposition of the modes
Table I. Values of $Z_{\text {eff }}$ for PDADMAC dissolved in different solvent mixtures

\begin{tabular}{|c|c|c|c|c|c|}
\hline \multirow{2}{*}{$\begin{array}{c}\text { Solvent } \\
c_{\mathbf{P}}\end{array}$} & \multicolumn{4}{|c|}{$\mathrm{NaCl}$} & \multirow{2}{*}{$\frac{\mathrm{Na}_{2} \mathrm{SO}_{4}}{8 \mathrm{~g} \mathrm{l}^{-1}}$} \\
\hline & $6 \mathrm{gl}^{-1}$ & $8 \mathrm{gl}^{-1}$ & $10 \mathrm{gl}^{-1}$ & $30 \mathrm{gl}^{-1}$ & \\
\hline Water & & & 19 & & 14.5 \\
\hline $25 \%$ Methanol & 8 & 8 & 9 & 11 & 7.5 \\
\hline $50 \%$ Methanol & & & 4.5 & & \\
\hline $25 \%$ Ethanol & & & 3.6 & & 3.5 \\
\hline
\end{tabular}
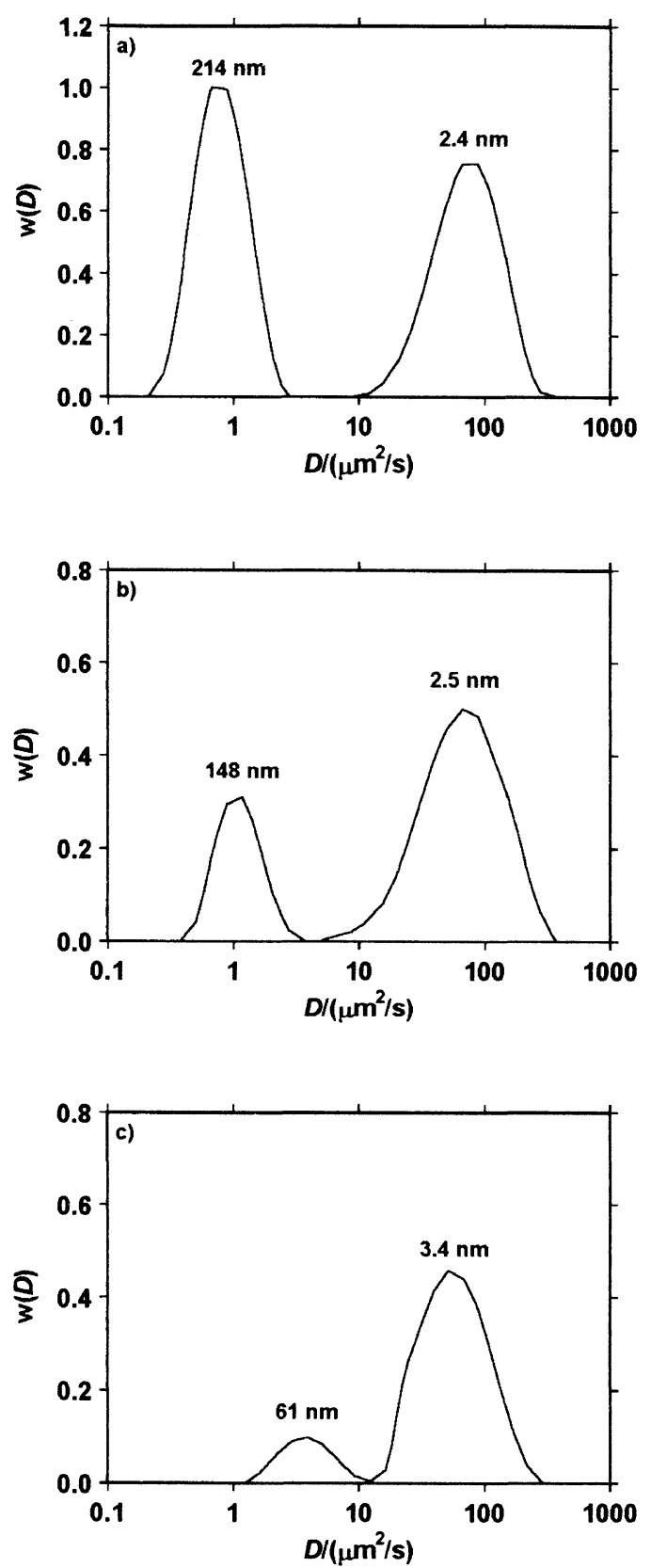

Figure 15. Diffusion coefficient distributions, $w(D)$, for PDADMAC dissolved in a mixture of methanol and water at $c_{\mathrm{P}}=8 \mathrm{gl}^{-1}$ and $c_{\mathrm{NaCl}}=10^{-3} \mathrm{M}$ where $x_{\text {methanol }}$ is a) $25 \mathrm{~mol} \%$, b) $50 \mathrm{~mol} \%$, and c) $75 \mathrm{~mol} \%$.

depends on $c_{\mathrm{P}}$. If $c_{\mathrm{P}}$ is $30 \mathrm{gl}^{-1}$ we have always three peaks. $\xi_{\mathrm{s}}$ becomes smaller with $c_{\mathrm{NaCl}}$ but it does not vanish as in the case for small $c_{\mathrm{P}}$ (see Figure 14). It is also seen that $\xi_{\mathrm{h}}$ increases significantly with $c_{\mathrm{NaCl}}$. That is, our speculation is right. The blob diffusion can be 

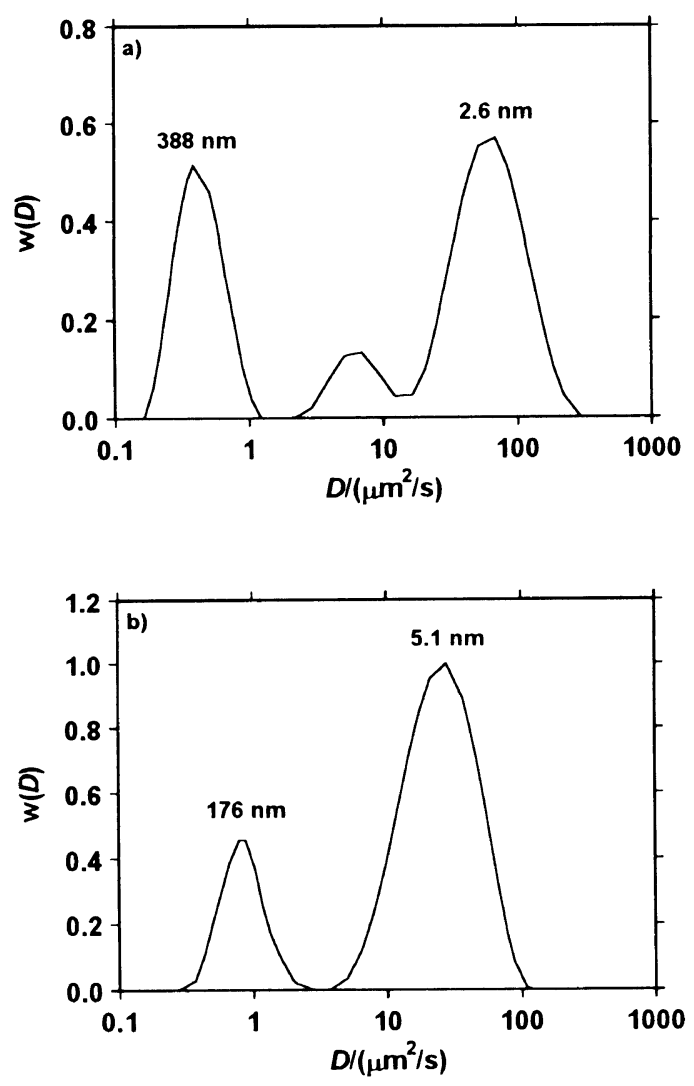

Figure 16. Diffusion coefficient distributions, $w(D)$, for PDADMAC dissolved in a mixture of $25 \mathrm{~mol} \%$ methanol and $75 \mathrm{~mol} \%$ water at $c_{\mathrm{P}}=8 \mathrm{gl}^{-1}$ where $c_{\mathrm{Na}_{2} \mathrm{SO}_{4}}$ is a) $3 \times 10^{-3} \mathrm{M}$ and b) $10^{-2} \mathrm{M}$.

interpreted by the coupled mode theory (eq 5). Some values of $Z_{\text {eff }}$, which is the effective charge of a polyion blob, are listed in Table I. We see that $Z_{\text {eff }}$ is more smaller the more organic the solvent is, while the influence of the counterion valence can be neglected.

Another interesting question is what happens when the alcoholic content of the solvent is increased. The system graphed in Figure 15 is PDADMAC, $c_{\mathrm{P}}=8 \mathrm{~g} \mathrm{l}^{-1}$, and $c_{\mathrm{NaCl}}=10^{-3} \mathrm{M}$, where $x_{\text {methanol }}$ is varied from $25 \%$ to $75 \%$. Above this mark PDADMAC is no longer soluble. Again we observe only two peaks, the cluster mode peak and the overlapping peak of the heterogeneity and the blob mode. The amplitude and the correlation length of the cluster mode decreases continually with increasing methanolic content. Since the same trend is observed for ethanol and propanol this suggests that alcohol destabilizes a cluster. However, there are differences. At a given salt and at a given polyion concentration the amplitude is largest for propanol and smallest for methanol. For instance, at $c_{\mathrm{NaCl}}=10^{-2} \mathrm{M}$ we find for methanol amplitude cluster-peak $/$ amplitude $_{\text {hetero-fast-peak }}=$ $1: 1$, for ethanol $2: 1$, and for propanol $2.5: 1$.

Of interest is also the influence of the type of the salt used. For this propose we inspect Figure 16 where $\mathrm{NaCl}$ is exchanged by $\mathrm{Na}_{2} \mathrm{SO}_{4}$, while $x_{\text {methanol }}=25 \%, c_{\mathrm{P}}=$ $8 \mathrm{gl}^{-1}$, and $T=25^{\circ} \mathrm{C}$ as before. We observe two effects: (1) the decomposition of the peaks becomes better with decreasing salt concentration and (2) the cluster correlation length, $\xi_{S}$, of $\mathrm{Na}_{2} \mathrm{SO}_{4}$ is larger than that of $\mathrm{NaCl}$. $\mathrm{SO}_{4}^{2-}$ bears two charges and $\mathrm{Cl}$ only one. It is therefore thinkable that $\mathrm{SO}_{4}^{2-}$ can connect two polyion
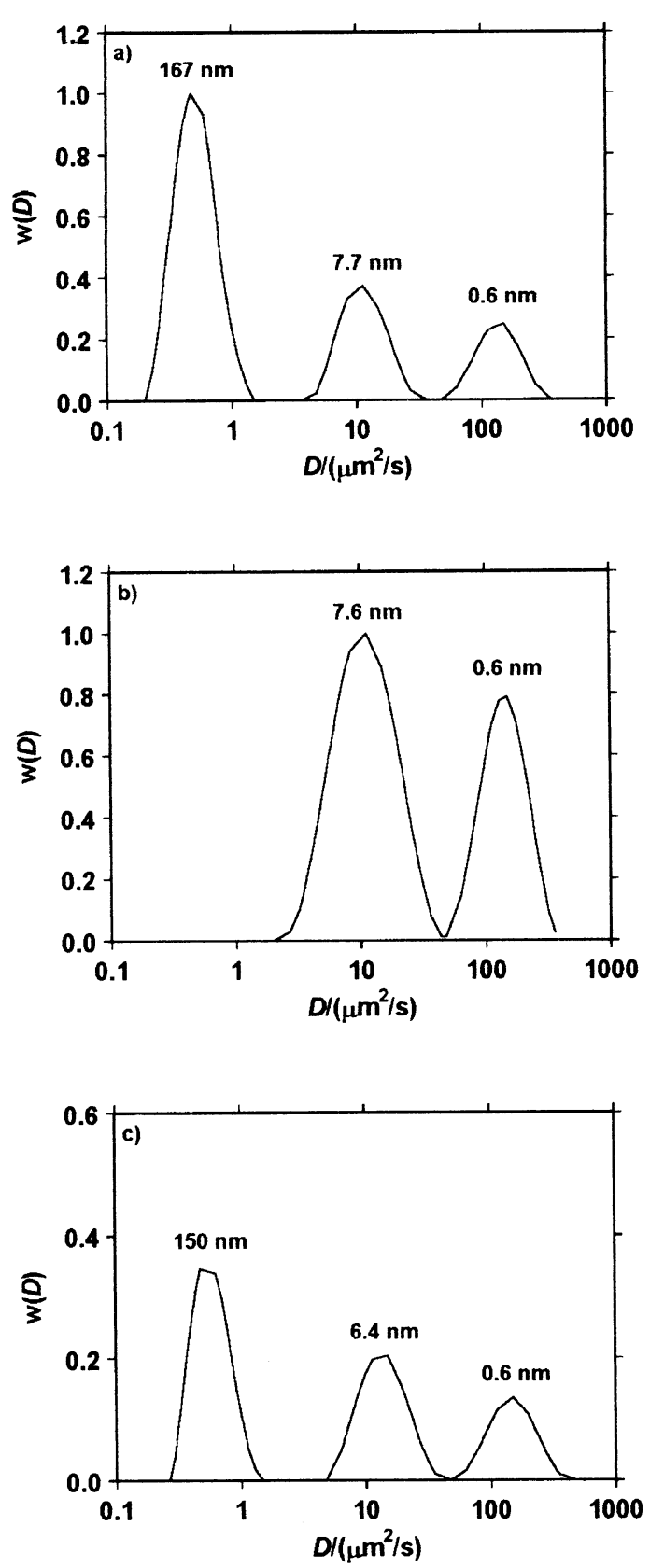

Figure 17. Diffusion coefficient distributions, $w(D)$, for PDADMAC dissolved in a mixture of $25 \mathrm{~mol} \% n$-propanol and $75 \mathrm{~mol} \%$ water at $c_{\mathrm{P}}=8 \mathrm{~g}^{-1}$ and $c_{\mathrm{NaCl}}=7.5 \times 10^{-3} \mathrm{M}$. a) presents the situation for $t=0$; b) is the result for $t=0$ after centrifugation; and c) is the situation when we wait again $t=4$ days.

chains to form a salt bridge. Such bridges would stabilizes a cluster so that larger structures may be built while in the presence of $\mathrm{NaCl}$ such salt bridges are rather unlikely.

Finally, we come to our last experiment. According to Reed et al. $^{31}$ the slow diffusive mode can be eliminated by filtration through a filter with an appropriate small enough pore size. We have tested this idea, not by filtration but by centrifugation with a laborfuge at 15000 $\min ^{-1}$ for 4 hours. Typical results are shown in Figure 17 for the system $75 \%$ water, $25 \% n$-propanol, $c_{\mathrm{P}}=$ $8 \mathrm{gl}^{-1}$, and $c_{\mathrm{NaCl}}=7.5 \times 10^{-3} \mathrm{M}$. Before centrifugation there are three diffusion modes which are separated quite well. The left peak with $\xi_{\mathrm{s}}=167 \mathrm{~nm}$ is the slow mode. This peak vanishes during centrifugation. This is in accord with Reeds idea, but if we wait 4 days the slow 
mode is back again and its dimension is nearly as large as before. This indicates that the so called slow mode is real and that there is an ordering process in the solution which depends on time and can be destroyed by outer forces such as centrifugal forces. This ordering process goes rather slowly. For water/propanol the ordering has become finished after 21 days. Then all three correlation lengths are as large as at the beginning.

\section{CONCLUSIONS}

We have performed dynamic light scattering measurements on PDADMAC dissolved in water/alcoholic mixtures varying the type of the added salt, the salt concentration, and the polyion concentration. The analysis of the data was done using two different methods, the old conventional one and the new multiple tau technique. The results obtained by the old method are in line with other similar studies. They may be interpreted by the theory of Schmitz and Ramsay. ${ }^{43}$ According to these authors it is suitable to characterize polyion diffusion by the ratio of polyion to salt concentration $\delta=c_{\mathrm{P}} / c_{\mathbf{S}}$. There exist three regimes.

The regime $\delta \ll 1$ is the dilute lattice regime. Usually, only one diffusion process is observed, the fast mode. The diffusion coefficient depends on the wave vector $q$, i.e., on the intermacromolecular structure factor $S(q)$. This proposes that $D_{\text {fast }}$ is a cooperative diffusion coefficient and that there is a spatial ordering of the polyion domains.

The second regime is the transition region where $\delta \approx 1$. Here, the slow diffusive process gradually appears. The exact onset of the splitting occurs at $\delta^{*}=(1 / k)\left(c_{\mathrm{p}} / c_{\mathrm{S}}\right)$, where the value of the constant $k$ depends strongly on the ability of the correlator used to separate the two modes. For a $1: 1$ salt according to Drifford and Dalbiez ${ }^{2}$ $k$ should be $2 \xi$ for PDADMAC/water and $12 \xi$ for PDADMAC $/ 25 \%$ methanol $/ 75 \%$ water. Our data are in agreement with these predictions. If, on the other hand, the counterions are bivalent or if the alcoholic content is very large, there is no diffusion splitting. Thus, it seems that $k$ depends not only on $\xi$ and the counterion valence, $z$, but additionally on a further unknown parameter.

The third regime is the semidilute regime, where $\delta \gg 1$. In most cases, one observes both a fast and as well a slow mode. This is the case for the systems PDADMAC/ $25 \%$ methanol $/ 75 \%$ water and $25 \%$ ethanol $/ 75 \%$ water. In both cases, $D_{\text {fast }}$ is independent on $\delta$, while $D_{\text {slow }}$ decreases slightly with increasing $\delta$. The concentration dependence of the fast diffusive mode can be attributed to the Nernst-Hartley diffusion ${ }^{11,45}$ of polyions. Scaling theory ${ }^{44}$ predicts $D_{\mathrm{fast}} \approx c_{\mathrm{P}}^{3 / 4}$ for solutions with added salt, but we cannot verify it. For PDADMAC $D_{\text {fast }}$ increases sigmoidaly from its dilute lattice regime to its plateau value in the semidilute regime.

The nature of the slow diffusion mode is not yet completely elucidated. We believe that the picture given by Schmitz and Ramsay ${ }^{43}$ is quite helpful. They suggest that at $\delta<1$ each polyion chain is surrounded only by its own ion cloud, while at $\delta \geq 1$, the ion clouds of neighboring polyion chains begin to overlap. The small ions in the overlapping regions cause forces that leads to a kind of binding between some neighboring polyions

Polym. J., Vol. 29, No. 10, 1997 so that a domain (cluster) of polyions is built whose polyion concentration is somewhat larger than in the residual solution. These domains are not stabile, they will randomly created and destroyed so that their dimensions and locations fluctuates (diffuse) with time.

A similar model was proposed by Fulton. ${ }^{46,47} \mathrm{He}$ postulated that long-range correlation's between fluctuating dipoles persist over long periods of time. At high salt concentrations the polyion charges are nearly totally screened by the small ions so that the correlations and electrostatic interactions become destroyed.

The results arrived by the multiple tau technique are significantly different from those obtained by the conventional method. There are not two diffusive modes but three modes, a cluster mode, a heterogeneity mode, and a blob mode. These modes are always present simultaneously. There is no diffusion splitting at $\delta \approx 1$ or at $c_{\mathrm{S}}^{*}$ as proposed by Drifford and Dalbiez. ${ }^{2}$ The decomposition of the three modes is, however, a problem. In most cases, the distributions of the heterogeneity and the blob mode overlap so that the corresponding peaks run together and the experimentator observes totally only two peaks. Best separation is achieved at high polyion concentration and in water/alcoholic mixtures with a $2: 1$ salt. For small salt concentrations we postulate long-range electrostatic interpolyion correlations over long periods of time, while at higher salt concentrations the polyion charges become more and more screened by the small ions so that the electrostatic binding (repulsion) between neighboring polyions become destroyed. As a consequence, the cluster correlation length decreases with increasing salt concentration. The same effect is observed when $c_{\text {salt }}$ is constant and the alcoholic content is increased. This is understandable since the degree of polyion dissociation is more smaller the more organic the solvent is. The heterogeneity mode is new. As far as we know it was never observed before. It describes the motion of partitions of polyion chains or whole chains which are not part of a polyion cluster. Their dimensions are of the order of $20 \mathrm{~nm}$ but can be also smaller so that a distinction from the blob mode becomes diffucult if not impossible. The blob mode itself can be interpreted as the coupled diffusion of a polyion blob with its ionic atmosphere. The poorer the quality of the solvent is the larger is the number of segments building a blob.

We have performed our measurements in the semidilute polyion region, that is, there may be a few polyion entanglements. The number and the stability of the entanglement points depends on the temperature, the salt concentration, and the solvent composition. They are randomly created and destroyed at random places in the solution. This fluctuations may induce the building of the cluster regions, but they may also destroy them.

Finally, let us make a critical comment. The model presented here is only a proposal. We have used only one method, namely dynamic light scattering. Other experimental techniques are necessary to support the results and to come to a more realistic model. There are many open and unsolved problems. We know nothing about the exact structure of the clusters and there is a lack of theory that describes the effects observed. That is, there remains a wide field of work for future studies. 
Acknowledgments. Support for this work was from "Der Fonds der Chemischen Industrie". We thank also Prof. M. D. Lechner for provision and making his laboratory facilities available. Finally, we return warmest thanks to our parents for sustained interest and encouragement.

\section{REFERENCES}

1. S. C. Lin, W. I. Lee, and J. M. Schurr, Biopolymers, 17, 1041 (1978).

2. M. Drifford and J. P. Dalbiez, Biopolymers, 24, 1501 (1985).

3. J. M. Schurr and K. S. Schmitz, Annu. Rev. Phys. Chem., 37, 271 (1986).

4. B. Chu and T. Nose, Macromolecules, 13, 122 (1980).

5. C. H. Wang, J. Chem. Phys., 95, 3788 (1991).

6. K. S. Schmitz and M. Lu, Biopolymers, 23, 797 (1984).

7. T. Nicolai and M. Mandel, Macromolecules, 22, 2348 (1989).

8. A. Patkowski, E. Gulari, and B. Chu, J. Chem. Phys., 73, 4178 (1980).

9. K. S. Schmitz and M. Lu, Proc. Natl. Acad. Sci. U.S.A., 80, 425 (1983)

10. R. S. Koene and M. Mandel, J. Mol. Biol., 196, 220 (1983).

11. M. Drifford and J. P. Dalbiez, J. Phys. Chem., 88, 5368 (1984).

12. M. Drifford and J. P. Dalbiez, J. Phys. Lett., 46, L-311 (1985).

13. K. S. Schmitz, M. Lu, and J. Gauntt, J. Chem. Phys., 78, 5059 (1983).

14. P. Mathiez, C. Mouttet, and G. Weisbuch, Biopolymers, 20, 2381 (1981)

15. K. S. Schmitz and J. Yu, Macromolecules, 21, 484 (1988).

16. M. Sedlak, C. Konak, P. Stepanek, and J. Jakes, Polymer, 28, 873 (1987).

17. S. Förster, M. Schmidt, and M. Antonietti, Polymer, 31, 781 (1990).

18. P. Tivant, P. Turq, M. Drifford, H. Magdelenat, and R. Menez, Biopolymers, 22, 643 (1983).

19. M. Sedlak and E. J. Amis, J. Chem. Phys., 96, 817 (1992).

20. J. Shibata and J. M. Schurr, Biopolymers, 18, 1831 (1979).
21. N. B. Martin, J. B. Tripp, J. H. Shibata, and J. M. Schurr, Biopolymers, 18, 2127 (1979).

22. K. Zero and B. R. Ware, J. Chem. Phys., 80, 1610 (1984).

23. J. P. Wilcoxon and J. M. Schurr, J. Chem. Phys., 78, 3354 (1983)

24. K. S. Schmitz and D. J. Ramsay, Biopolymers, 24, 1247 (1985).

25. L. Wang and V. A. Bloomfield, Macromolecules, 24, 5791 (1991).

26. N. Ise, T. Okubo, K. Yamamoto, H. Matsuoka, H. Kawai, T. Hashimoto, and M. Fujimura, J. Chem. Phys., 78, 541 (1983).

27. N. Ise, T. Okubo, K. Yamamoto, H. Matsuoka, H. Kawai, T. Hashimoto, M. Fujimura, and Y. Hiragi, J. Am. Chem. Soc., 102, 7901 (1980).

28. M. Levij, J. De Bleijser, and J. C. Leyte, Chem. Phys. Lett., 83, 183 (1981).

29. D. J. Ramsay and K. S. Schmitz, Macromolecules, 18, 2422 (1985).

30. R. M. Peitzsch, M. J. Burt, and W. F. Reed, Macromolecules, 25, 806 (1992).

31. W. F. Reed, S. Ghosh, G. Medjhadi, and J. Francois, Macromolecules, 24, 6189 (1991).

32. T. Nicolai and M. Mandel, Macromolecules, 22, 438 (1989)

33. M. Sedlak, Macromolecules, 26, 1158 (1993).

34. J. Xia, P. L. Dubin, T. Izumi, M. Hirata, and E. Kokufuta, J. Polym. Sci., Part B, Polym. Phys., 34, 497 (1996).

35. J. M. Schurr, CRC Crit. Rev. Biochem., 4, 371 (1977).

36. H. Z. Cummins and E. R. Pike, "Photon Correlation Spectroscopy," Plenum Press, New York, N.Y., 1977.

37. D. E. Koppel, J. Chem. Phys., 57, 4814 (1972).

38. N. Ostrowsky, D. Sonette, P. Parker, and E. R. Pike, Exponential Sampling Technique, Optica Acta, 28, 1059 (1981).

39. S. W. Provencher, CONTIN, Computer Physics, Communication, 27, 213-242 (1982).

40. G. S. Manning, Rev. Biophys., 11, 179 (1978).

41. K. W. Rhee and B. R. Ware, J. Chem. Phys., 78, 3349 (1983).

42. M. E. Ferrari and V. A. Bloomfield, Macromolecules, 25, 5266 (1992).

43. K. S. Schmitz and D. J. Ramsay, Macromolecules, 18, 933 (1985).

44. F. Grüner, W. P. Lehman, H. Fahlbusch, and R. Weber, J. Phys. (A) Math. Gen., 14, L-307 (1981).

45. R. S. Koene and M. Mandel, Macromolecules, 16, 688 (1983).

46. R. L. Fulton, J. Chem. Phys., 68, 3089 (1978).

47. R. L. Fulton, J. Chem. Phys., 78, 6865 (1983). 